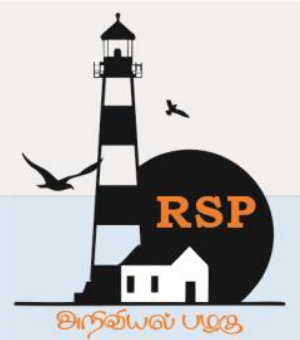

INTERNATIONAL RESEARCH JOURNAL ON

e-ISSN : 2582 - 4376 ADVANCED SCIENCE HUB Open Access

RSP SCIENCE HUB

(The Hub of Research Ideas)

Available online at www.rspsciencehub.com

\title{
Myoelectric Potential Visualization Using Butterworth Band pass Filter
}

Puviyarasan. $S^{1}$, Muthukumaran. $S^{2}$, Pratheen raj. $B^{3}$,

${ }_{1,2,3}^{1,2 e s e a r c h ~ i n t e r n, ~ K E D S G R O U P S ~ R e s e a r c h ~ a n d ~ D e v e l o p m e n t, ~ C o i m b a t o r e . ~}$

${ }^{1,2,3} 3$ rd year Student, Department of Biomedical Engineering, Salem college of Engineering and Technology, Tamilnadu, India .

puviarasan161@gmail.com ${ }^{1}$, sivakumaranpml27@gmail.com ${ }^{2}$,pratheenrajb@gmail.com ${ }^{3}$

\begin{abstract}
Electrical revelation of neuromuscular information transmission generated in muscles contraction and relaxation is known as EMG signals. Electromyography $(E M G)$ is real-time based method to assess and observe a series of electrical signals that expressed from body muscular cells. In recent researchers had provided many systems for monitoring myoelectric signals (EMG) to identify various abnormalities such as EMG, Microcontroller sensors technology, EMG software signal processor (SPU), PWM method, capacitive sensing method, UML method, EIM method, ADU integration, MC sensors method, Human computer interfacing (HCI) technology and Functional electrical stimulation (FES). This paper proposes a system to implement a wireless transformation technology for monitoring the electrical potential from muscles to identify internal injuries, blood clots, muscle cramps, muscle fatigue, muscle contraction, limb stiffness, and immobility. In this paper, electrical signals acquired from the muscles are detected using EMG sensors. These occurring signals transmitted by using wireless method and processed by using MAT Lab tool. This technique makes our system more unique from the previous methodologies. Finally accurate EMG signals are displayed graphically. Thus the expected configuration result will be with an accuracy of $98.74 \%$, mean specificity of $99 \%$ and with a mean sensitivity of $96.58 \%$. So the error occurrence will be Approximately $0.5 \%$ and also system is low cost, electrical safety, low power consumption, and can identify muscle disorders through observed abnormal EMGrange.
\end{abstract}

Keywords: Myoelectric potential, EMG sensors, Transmitter, Receiver, MAT lab tool, Butter worth band pass filter, amplifier

\section{Introduction}

One of the Electro diagnostic techniques is Electromyography (EMG), which is used to evaluate the muscle activities and detect electrical potential of muscular cells. Detect medical abnormalities, activation level, and other measurements using EMG signal analysis. Human arm involves in many working activities, so arms and muscles may have fatigue and pain. We have Developed Myoelectric potential monitoring System. From literature survey, the diagnosis and comparison of different muscular disorders are detected using Human Computer Interfacing (HCI), Non-invasive detection of neuromuscular diseases applying the technique of Electrical Impedance Myography (EIM), indicate the feasibility and efficiency of EIM. Design of cMOS transistors operating in low level version shown to offer the high level energy here the electrode-body interfaces offered for acquisition, electrode-tissue impedance measurement and optical Biosignalsensing. Monitoring of muscular activation for rehabilitation, muscle activation plans, prosthetics control, myoelectric bracelets, and identification of possible pathologies using Myoelectric interfaces was performed. In this paper we designed a system to monitor the 
electrical signals of the muscles in the body through electromyography sensors. This system is specially designed to diagnose internal injuries, blood clots, muscle cramps, muscle fatigue, muscle contraction, limb stiffness, and immobility. It can be used by patients, children, exercisers and the physically challenged. Our proposed system was divided it into two parts .The first part is Sensing part that was fixed on the body. Another part is monitoring part, here we use an EMG sensor to detect electrical potential from muscle activities and also two types of electrodes are used such as Intramuscular electrodes and surface electrodes in medical Instrumentation. Especially here we used a Myoware Muscle Sensor.

The EMG sensor collects the signals and then converts it into an electrical signal. Electrical signals arrive at the monitor via a wifi router and then the signals are received by the wifi receiver and fed to the pre amplifier, where the frequency of the signal is slightly increased and noise where removed by using Band pass filter. The frequency of the EMG signal is highly increased by using an Instrumentation amplifier, finally The Amplified signals pre-processed and displayed by MATLAB.

\section{Background}

Since the innovation of Muscle monitoring by Edgardo L.Mercado Medina, Design of an Electronic System for Monitoring Muscle Activity. This system is a starting technique to observe the muscle activities. This Project had given more importance to design electronic circuits for monitoring electrical signals of muscles. In this project, neuro-muscular system studied by using Electromyograpy (EMG). This system compressed of two essential elements. First element is electrodes, that convert the potential current in the body muscles. Next one is electronic circuits, which is used for signal conditioning and output observations. Electrodes used in the previous studies might be invasive or non - invasive. In this paper, they selected non-invasive type electrodes that were placed in the surface of the body. Thus the EMG signals produced by transporting of ions through cell membranes of muscle fibers, thus the amplitude of common EMG signals will be in the range of $0.1 \mathrm{mV}$ to $5 \mathrm{mV}$ and the frequency of sEMG signals go from 0 to $450 \mathrm{~Hz}$. In the low frequencies, Most of energy of the signals located.
The Journal of Electromyography requires the corner frequency of $10 \mathrm{~Hz}$. In this paper the author recommend a method using high frequencies that include intense physical activities. The EMG signals uses additional applications such as biomechanic analysis of movement, rehabilitation and monitoring system for muscle activity in sports.

In 2008, Young.et.al. developed a wireless EMG micro system to performed the myo-electric control of aprosthesis

B. Khalid, et.al .In this system he identified the field of muscle monitoring of a normal person by varying load as a application of EMG signals. This helps us to get signals from patient's body very efficiently. EMG technique and reveal the methodologies for analying the occurring signal. This paper study EMG signal detection, decomposition, process and discovery of the disadvantages in one method leads to other improving methods. Bernabe Rodriguez Tapia . Myoelectric Interfaces referred the Human Computer Interaction field (HCI). It's a next level improvement of Electromyograpy technique. In this paper, Signals acquisition consists of four main stages: [1] Signal collection, [2] Signal amplification,[3]Signal filtering,[4] Analog to digital converters. In this technique they improved the parameters for implementation of EMG signals. SohmyungHa [5] Integrated circuits and Electrode interfaces, it is a advanced tremendously over the year make a huge impact on medical diagnostics and personal healthcare. This innovation was designed by using semiconductor technologies. In this paper, we designed a system using EMG sensor technology to evaluate themuscle activities and detect electrical potential of muscular cells by EMG signals acquired through EMG sensor. This system is designed to diagnose internal injuries, blood clots, muscle cramps, muscle fatigue, muscle contraction, limb stiffness and immobility. The EMG sensors was inexpensive, easy to wear and we modified that to easily attached patients muscles. In this system we developed a wireless methodology. We use wireless transceiver technical device for transmitting EMG signals to visualization. Most important part is MAT Lab processing unit, that is used for filtering, Amplifying, pre-processing. We designed this system mostly using software algorithms for observed the accurate EMG signals. 


\section{Literature survey}

Serge H. Roy proposed the system for physical activity by using body worn sensors. This system achieved 'FIM related activities, which is used for evaluate the action mobility of patients and also identify the disorders of motor units. This study referred the movement of muscular activity during walking patterns.

CharnLoong described the medical system for medical assessment of abnormalities and treatment. This EMG system using for Remote Healthcare Monitoring(RHM). This system build by using biosensors.

Li Yuan derived the IOT techniques for updating human based terminals. C.Pylatiuk make a system for clinical use. In this system, they used four different polymers for EMG detecting and different clinical applications.

\section{Block Diagram}

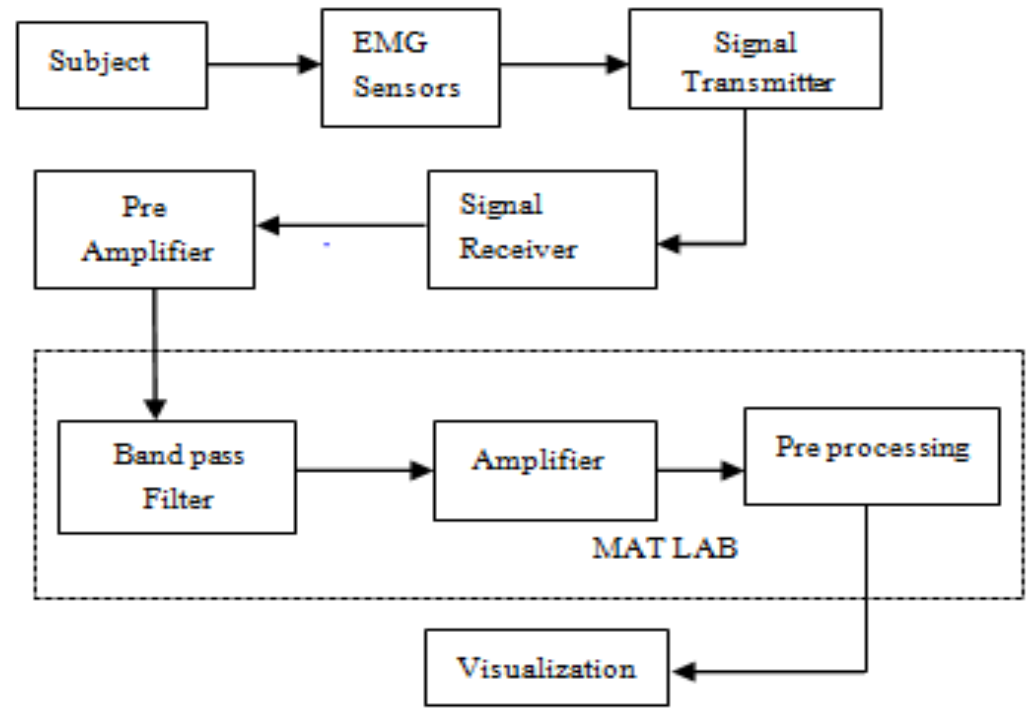

Fig.1. Block Diagram of the System

\section{Working}

The EMG signals of muscle fatigue, activities from patient's body collected using latest EMG sensors. We use EMG sensors with Arduino, Myoware versions. This whole muscle activity process starts from brain. Muscle activities also related with neural activity in the motor cortex signals to the spinal cord. The Neuro impulse is conveyed to the muscle part via motor neurons. In this process motor neurons directly connected to the muscle fibers, release of calcium ions within the muscle and produce mechanical changes.

\subsection{EMG sensing \& transmitting process:}

We use surface Electromyography sensing electrodes (sEMG) for detect electrical potential of muscular activities from muscle fibers. The Signal sensing process initiated with EMG sensor placement. EMG sensors will sense the Muscle signals. Therangeofasignalisabout $0.20 \mathrm{Vto}$

$0.40 \mathrm{~V}$ for biceps, limb and muscle fibers. No patches need for these sensor. We designed this system with a transmitter attached to EMG sensor this is called as attachment part attachment part. This transmitter will transmit the signals from the range of $40 \mathrm{~Hz}$ to $450 \mathrm{~Hz}$ and its bandwidth range will be $25--500 \mathrm{~Hz}$.Next is stable part for viewing output.

\subsection{Receiving Process}

We use the wireless transceiver module for collecting the EMG signals from EMG sensors. It is NRF905 work band wireless transceiver it has 170 channel numbers and it is used for the transmission and receiving process. To perform this process this transceiver uses a PLL frequency stabilization method. Its maximum is about +10.5 $\mathrm{dbm}$ depends upon the transmitting signal range. Its receiving frequency capacity is $120 \mathrm{~Hz}$ to 915 $\mathrm{MHz}$.

\subsection{Pre amplifying process:}

We use PAM8403 based preamplifying circuit, it is used to amplify the signals from 1- $200 \mathrm{kHZ}$. In this process the value repeating voltage is about 1 to $1.5 \mathrm{~V}$. This preamplifier is used to increase 
small amount of gain level (upto $560 \mathrm{~dB}$ ), it is used for the signal processing. These pre-amplified signals are preceded with the filtering Unit.

\subsection{Signal processing:}

We use MAT lab for signal processing. This software programming is a modified technology to replace the electrical circuits. Before the signal processing, the EMG signals should be filtered and amplified for visualization.

\subsubsection{Filtering process:}

Mostly four types of filters are used in electrical system such as low pass filter, high pass filter, band pass filter and band stop filter. In the modern programming technique the Butterworth filters are used for signal conditioning process. Butterworth filter also classified into four types such as Low pass Butterworth filter, High pass Butterworth filter, Band pass Butterworth filter and Band stop Butterworth filter. In this project, we use Band pass Butterworth filter it is a 20-th order Butterworth band pass filter with a lower cutoff frequency is about $500 \mathrm{~Hz}$ and its higher cutoff frequency is about $560 \mathrm{~Hz}$. We can change the low and high cutoff frequencies depends upon the input of EMG signals. In this system we use MAT lab to perform filtering process using "designfilt". [b,a] = butter (n,Wn,ftype) is a common syntax code for designing low, high, band pass and band stop butterworth filters depends upon its applications. The Bandpass filter use the 'bandpass' hint for "ftype". The input arguments are $\mathrm{n}$ and $\mathrm{Wn}, \mathrm{n}$ represents one half order of the filter, $\mathrm{Wn}$ represents the cutoff frequency. Incase $\mathrm{Wn}$ is the two element vector [W1,W2], W1 is the low cut off frequency and W2 is the high cut off frequency in digital form it is represented as $\mathrm{W} 1=0$ andW2 $=1$.

The transfer function coefficients $(b, a)$ is used in the output arguments. The row vector of length is

Table.1.Myoelectric potential for identification $\mathrm{n}+1$ for low pass and high pass filters as well as the length of $2 n+1$ for band pass and band stop filters.

Finally we filtered the EMG signals without noises using Band pass Butterworth filter with the required frequencies.

\subsubsection{Signal Amplification:}

For Electrical applications, mostly Instrumentation amplifier and operational amplifier are used. In instrumentation amplifier the voltage gain will be higher. So instrumentation amplifier is commonly used for many systems. In this system we used software coding amplifier this amplifier does not require any circuits, semiconductor devices, because the amplification is performed by using MAT Lab. In MAT lab, amplifier is a 2 port RF circuit object and we can use 'rfbudget' and 'circuit' object elements for EMG signal amplification. amp =amplifier ( Name,Value) is the common syntax of amplifier coding.

\subsection{Properties:}

Name of the amplifier, Gain values, Noise figure (NF), Output third order intercept (OIP3), Input Impedance (Zin), Output impedance (Zout), NumPorts( number of ports), Number of portterminals (Terminals), these are the basic properties of amplifier in MAT Lab. In this paper we use Instrumentation amplifier focusing its features. The gain rangeincreased up to $120 \mathrm{~dB}$ to $140 \mathrm{~dB}$. Noise figure value is Zero. We using impedance matching for get maximum output, so Input impedance and output impedance are equal and maximum value will be up to $1 \mathrm{G} \mathrm{ohm}$.

Finally the processed EMG signals fed to the visualization part. Through monitoring system we represents the signals both Analog and digital form for real time applications.

\begin{tabular}{|c|c|c|}
\hline Potential & Normal value & Abnormal value \\
\hline Resting position & $\begin{array}{l}-70 \text { to } \\
-90 \mathrm{mV}\end{array}$ & NIL \\
\hline Contraction and relaxation & $\begin{array}{c}-50 \text { to } \\
-55 \mathrm{mV}\end{array}$ & 450 to $780 \mathrm{mV}$ \\
\hline
\end{tabular}


Table.2. Comparison table for result Accuracy

\begin{tabular}{|c|c|c|c|}
\hline Systems & Technique & $\begin{array}{c}\text { Problem } \\
\text { statement }\end{array}$ & Result accuracy \\
\hline $\begin{array}{c}\text { Muscle Activity in } \\
\text { Weight- lifting }\end{array}$ & $\begin{array}{c}\text { Real time human } \\
\text { computer interfacing. }\end{array}$ & $\begin{array}{c}\text { Diagnosis and } \\
\text { comparison }\end{array}$ & $55 \%$ \\
\hline $\begin{array}{c}\text { Non- invasive } \\
\text { Monitoring. }\end{array}$ & Body-electrode interface, & $\begin{array}{c}\text { Muscle monitoring } \\
\text { systems }\end{array}$ & $76 \%$ \\
\hline $\begin{array}{c}\text { Muscle } \\
\text { Monitoring system } \\
\text { varying load) }\end{array}$ & $\begin{array}{c}\text { Electromyography, EMG } \\
\text { signal sensors. }\end{array}$ & $\begin{array}{c}\text { Diagnosis and } \\
\text { comparison }\end{array}$ & $73 \%$ \\
\hline $\begin{array}{c}\text { Electrical } \\
\text { Impedance } \\
\text { Myography }\end{array}$ & $\begin{array}{c}\text { Optimized electrode } \\
\text { configurations. }\end{array}$ & $\begin{array}{c}\text { Non- Invasive } \\
\text { detection of } \\
\text { neuromuscular } \\
\text { diseases. }\end{array}$ & $79 \%$ \\
\hline $\begin{array}{c}\text { Myoelectric } \\
\text { Interfaces }\end{array}$ & $\begin{array}{c}\text { EMG acquisition system, } \\
\text { myoelectric control. }\end{array}$ & $\begin{array}{c}\text { Monitoring of } \\
\text { muscular activation }\end{array}$ & $84 \%$ \\
\hline
\end{tabular}

In this paper, we use upgraded EMG sensors for collecting myolectric potential from the human body. And also use MAT Lab tool for result accuracy. In this study the result accuracy ranges of $98 \%$.

\section{Result}

The electrical potential of the muscle fibers are electrically inactive at rest position. During the contraction \& relaxation of the muscles and limbs nodes the electrical potential will be active. Thus the Measured EMG signal range will be initiated at 30 microvolt up to 30 milli volt depends on muscular activities. So EMG signals lies in the frequency range from 5 to $450 \mathrm{~Hz}$ and most dominant range will be 50 to $150 \mathrm{~Hz}$ and in damaged muscles the frequency range lies between $450 \mathrm{mVto} 780 \mathrm{mV}$, It is a abnormal range of EMG signals occurred from injured part of the muscular fibers. We can identify the muscle disorders using these abnormal values.

\section{Conclusion}

This study clearly delivered a system for identifying the range of electrical potential of muscle activities. It is enable to diagnosis of muscle injury and disorders through observed EMG signals. In this paper, the EMG signals collected from the body and transmitted using attached transmitter system, transmitted signals observed by the receiver. Finally EMG signals processed and displayed using MAT Lab. For EMG signal filtering, amplifying, preprocessing, we use MAT Lab. So this system low cost, electrical safety, low power consumption and as programmed circuits, give more accuracy outputs.

\section{Acknowledgement}

I wish to thank Ms.Mounikaasenthil and other mentors of KEDS GROUPS Research and Development for their constant support and effective training on writing this paper.

\section{References}

\section{Conference Proceedings}

[1].Li Yuan, Haikou, Junlin Chen * "Activity EMG Signal Identification Based on Radial Basis Function Neural Networks,"2017 IEEE.

[2].Nuradebah Burhan1*, Mohammad 'Afif Kasno2, RozaimiGhazali, "Feature Extraction of Surface Electromyography (sEMG) and Signal Processing Technique in Wavelet Transform: A Review,"2016 IEEE International Conference on Automatic Control and Intelligent Systems (I2CACIS), 22 October 2016, Shah Alam, Malaysia,2016 IEEE. 
[3].H.R. Khataee, T.N. MohdAris, M.N. Sulaiman,"An Agent-Based Model of Muscle Contraction Process as a Bio- Robotic Process,"2011 5th Malaysian Conference in Software Engineering,2011 IEEE.

[4].D. Zhang, Y. Matsuoka, W. Kong, U. Imtiaz, L. Bartolomeo, S. Cosentino, M. Zecca, S. Sessa, H. Ishii, and A. Takanishi, "Development of new muscle contraction sensor to replace sEMG for using in muscles analysis fields,"2014IEEE.

[5].CharnLoong Ng, MamunBin IbneReaz "A LowNoise Capacitive Electromy ography iMonitoring System for Remote Healthcare Applications,"2019 IEEE.

[6].Eisha B. Khalid, Afra Salman, Zeeshan Y. Khan, Muhammad Faris, Muhammad Danish Mujib, "Design and Development of Muscle Monitoring system to visualize and monitor the activity of Bicep Brachii with varying load,"2014IEEE.

[7].Edgardo L. Mercado-Medina*, Zulay D. Chavarro-Hernandez Juan A. DominguezJimenez*, and Sonia H. ContrerasOrtiz*,"Design of an Electronic Systemfor Monitoring Muscle Activity in Weightlifting,"2014IEEE.

[8].Bernabe Rodríguez-Tapia1,2, Israel Soto, Daniela M. Marínez1,And Norma Candolfi Arballo "Myoelectric Interfaces and Related Applications: Current State of EMG Signal Processing-A Systematic Review," 2020 IEEE.

[9].JING LUO 1, (Student Member, IEEE), CHAO LIU 2, (Senior Member, IEEE), AND CHENGUANG YANG3, (Senior Member, IEEE), "Estimation of EMG- Based Force Using Neural-Network-Based Approach," 2019IEEE.

[10].Ali Salman, Javaid Iqbal, UmerIzhar, Umar Shahbaz Khan, Nasir Rashid, "Optimized Circuit for EMG Signal Processing,"2014IEEE.

[11].Sumit A. Raurale, "Acquisition and Processing Real-time EMG signals for Prosthesis Active Hand Movements," 2014 IEEE.

[12].Bernabe Rodríguez-Tapia , Israel Soto, Daniela M. Martínez And Norma Candolfi Arballo "Myoelectric Interfaces and Related
Applications: Current State of EMG Signal Processing-A Systematic Review," 2020. IEEE.

[13].Richard B.Woodward , Sandra J. Shefelbine\& RaviVaidyanathan ,"Pervasive Monitoring of Motion and Muscle Activation: Inertial and Mechanomyography Fusion," 2017,IEEE.

[14].Sahil Gera T,George Tharion, Suresh Devasahayam *,Prashanth H Chalageri ,Navin B P,Raji Thomas "Electrical Stimulation and assessment of the induced force in the Denervated Muscle,"2019 IEEE.

[15].Jai Mangal,"A Smart Wear based Portable Health Monitoring System,"2020 IEEE.

[16].LI Shu-Ying and XIAO Xian-Jian,ZhouGengYua,"A High-voltage Circuit Breaker Contacts Temperature Monitoring System Based on Wireless Technology," 2015 IEEE.

[17]. D. Zhang, Y. Matsuoka, W. Kong, U. Imtiaz, L. Bartolomeo, S. Cosentino, M. Zecca, S. Sessa, H. Ishii,A. Takanishi,"Development of new muscle contraction sensor to replace sEMG for using in muscles analysis fields," 2017 IEEE.

[18].Bojan Milosevic, SimoncBenatti, ElisabettaFarella, "Design Challenges for Wearable EMG Applications," 2017IEEE.

[19].NecmettinSezginBatman, Turkey, "Analysis of EMG Signals in Aggressive and Normal Activities," The Scientific World Journal Volume 2012, Article ID 478952, the scientific World JOURNAL, Research, T.Arendt and J. Benito-Leon 2012.

[20].Jakub Emanuel Takosoglu, PawelAndrzel Laski, SlawomirBlasiak, "Determining the Static Characteristics of Pneumatic muscles," 2016 Measurement and controls. 